\title{
Limited Sampling Strategy to Predict AUC of the CYP3A Phenotyping Probe Midazolam in Adults: Application to Various Assay Techniques
}

\author{
Jooran S. Kim, PharmD, Anne N. Nafziger, MD, MHS, Shirley M. Tsunoda, PharmD, \\ Edna F. Choo, PhD, Daniel S. Streetman, PharmD, Angela D. M. Kashuba, PharmD, \\ Robert W. Kulawy, BS, Debra J. Beck, BS, Mario L. Rocci, Jr., PhD, Grant R. Wilkinson, PhD, \\ David J. Greenblatt, MD, and Joseph S. Bertino, Jr., PharmD
}

Midazolam clearance is used to phenotype hepatic CYP3A activity but requires multiple plasma samples following a single intravenous dose. The authors evaluated the use of a limited sampling scheme, using different assay techniques to determine the reproducibility of such a strategy in estimating midazolam AUC. Seventy-three healthy adults received midazolam as a single intravenous bolus dose. At least eight plasma samples were collected from each subject and were assayed using either LC/MS/MS or electron capture gas chromatography. Eleven subjects were randomly selected for the training set using stepwise linear regression to determine relationships between midazolam plasma concentrations and AUC. Validation of the predictive equations was done using the remaining 62 subjects. Mean percent error (MPE), mean absolute error (MAE), and root mean square error (RMSE) were calculated to determine bias and precision. Based on the training set, five models were generated with coefficients of determination ranging from 0.87 to 0.95. Validation showed that MPE, MAE, and RMSE values were acceptable for three of the models. Intrasubject reproducibility was good. In addition, training set data from one institution were able to predict data from the other two institutions using other assay techniques. Minimized plasma sampling may provide a simpler method for estimating midazolam AUC for CYP3A phenotyping. A limited sampling strategy is more convenient and cost-effective than standard sampling strategies and is applicable to more than one assay technique.

Journal of Clinical Pharmacology, 2002;42:376-382 C2002 the American College of Clinical Pharmacology
$\mathbf{T}$ he use of in vivo probe substrates is a widely accepted method of evaluating cytochrome P450 activity in individuals. ${ }^{1,2}$ By administering a suitable substrate for the enzyme in question, enzymatic activity

\footnotetext{
From the Clinical Pharmacology Research Center, Bassett Healthcare, Cooperstown, New York (Dr. Kim, Dr. Nafziger, Dr. Bertino); School of Pharmacy, Bouvé College of Health Sciences, Northeastern University, Boston (Dr. Tsunoda); Department of Pharmacology, Vanderbilt University, Nashville, Tennessee (Dr. Choo, Dr. Wilkinson); School of Pharmacy, University of Michigan, Ann Arbor, Michigan (Dr. Streetman); Department of Pharmacy, University of North Carolina at Chapel Hill, North Carolina (Dr. Kashuba); Oneida Research Services, Inc., Whitesboro, New York (Mr. Kulawy, Ms. Beck, Dr. Rocci); and Department of Pharmacology and Experimental Therapeutics, Tufts University School of Medicine, Boston (Dr. Greenblatt). Submitted for publication June 28, 2001; revised version accepted December 31, 2001. Address for reprints: Joseph S. Bertino, Jr., PharmD, Codirector, Clinical Pharmacology Research Center, Bassett Healthcare, One Atwell Road, Cooperstown, NY 13326-1394.
}

can be evaluated by comparing total or fractional clearance, metabolic ratios of parent compounds, and/or their metabolites in the plasma, urine, saliva or breath of an individual.

The plasma clearance of intravenously administered midazolam, a benzodiazepine used clinically for conscious sedation, has been used as an in vivo marker of hepatic CYP3A activity. Thummel and colleagues were the first to investigate the utility of midazolam as a phenotyping probe for CYP3A. ${ }^{3-5}$ Importantly, they observed a strong correlation between the systemic clearance of midazolam and in vitro hepatic CYP3A activity. This was the basis for the use of this pharmacokinetic parameter as a phenotypic measure of hepatic CYP3A activity. ${ }^{5,6}$

To calculate systemic clearance using midazolam, the area under the curve $\left(\mathrm{AUC}_{0-\infty}\right)$ of midazolam must be determined. This requires multiple plasma samples 
to be collected over a 6- to 8-hour period. Generally, at least eight plasma samples have been used to accurately determine midazolam $\mathrm{AUC}_{0-\infty}{ }^{3-5,7-9}$ The inconvenience and cost of extensive plasma sampling limit the utility of using intravenous midazolam as an easy and routine measure of CYP3A metabolic activity. Ideally, if fewer samples were used, the determination of midazolam $\mathrm{AUC}_{0-\infty}$ would be less expensive and simpler.

The limited sampling strategy (LSS) was first introduced in 1987 to minimize the number of plasma samples necessary to estimate AUC values of chemotherapeutic agents, immunosuppressive agents, antimicrobials, and other index substrates. ${ }^{10-17}$ This approach has proven to be an effective, validated tool in monitoring efficacy and toxicity of agents while minimizing the number of blood samples.

To date, no studies have applied the limited sampling strategy method to determine pharmacokinetic parameters for specific cytochrome $\mathrm{P} 450$ phenotyping agents. In addition, different assay methods are often used in phenotyping studies. These different assay methods may have different accuracy and precision performance; thus, if LSS is to be applied to phenotyping probes, assurance that the LSS methods are applicable to more than one assay method is important.

The objectives of this study were (1) to evaluate the use of minimized plasma sampling to determine the least number of plasma samples necessary to accurately estimate the $\mathrm{AUC}_{0-\infty}$ of midazolam following a single intravenous dose, (2) to validate the developed models for application with different assay techniques (LC/MS/MS and electron capture gas chromatography) at different institutions, and (3) to determine if the models give reproducible results in subjects undergoing repeat phenotyping measurements with midazolam.

\section{METHODS}

Data sets from three separate institutions were used in the study, and details of the original findings have been individually reported. ${ }^{6,8,18,19}$ This study was approved by the Institutional Review Board at Bassett Healthcare in Cooperstown, New York (Institution I); the Human Investigation Review Committee at the New England Medical Center and Tufts University in Boston (Institution II); and the Institutional Review Board-Health Sciences at Vanderbilt University (Institution III). Written informed consent was obtained from all study subjects.

\section{Subjects}

\section{Institution I}

Thirty-three healthy volunteers were enrolled. Baseline laboratory measurements (i.e., complete blood count, serum chemistries, urinalysis) and electrocardiogram were obtained prior to study participation. Each subject underwent a complete physical examination and medical history to ensure that previously described inclusion and exclusion criteria were met. ${ }^{8}$ Smokers and binge drinkers were excluded. Alcohol intake was limited to one drink (equivalent to one 12-ounce beer) per day, and no concomitant medications were allowed. Individuals with liver function tests (i.e., aspartate aminotransferase, alanine aminotransferase) greater than 1.5 times the upper limit of normal, total bilirubin greater than $1.5 \mathrm{mg} / \mathrm{dl}$, or serum creatinine values not within the normal range (0.6-1.2 $\mathrm{mg} / \mathrm{dl}$ for men; $0.5-1.0 \mathrm{mg} / \mathrm{dl}$ for women) were also excluded.

Subjects were administered intravenous midazolam $0.025 \mathrm{mg} / \mathrm{kg}$ (Versed: $2 \mathrm{mg} / \mathrm{ml}$ for injection, HoffmanLaRoche, Nutley, NJ) into an antecubital vein over 1 minute. Blood samples $(7 \mathrm{ml})$ were collected through an intravenous catheter (in the contralateral arm) into EDTA-containing tubes at predose and 5, 30, 60, 120, 240, 300, and 360 minutes following midazolam administration. Ten subjects had five repeated administrations of single intravenous boluses of midazolam at 2-week intervals. The blood samples were centrifuged; plasma was harvested and frozen at $-80^{\circ} \mathrm{C}$ until analysis.

\section{Institution II}

Data were obtained from 11 healthy nonsmokers who did not take any concomitant medications for the duration of the study. Medical histories, physical examinations, and blood work were obtained for screening.

Subjects received intravenous bolus midazolam 2 mg and had blood samples collected at predose and 15, $30,45,60,90,120,150,180,240,300,360$, and 480 minutes following midazolam administration.

\section{Institution III}

Twenty-nine healthy volunteers were enrolled. Subjects were nonsmokers who did not take any concomitant medications for the study duration. For screening purposes, medical histories and physical examinations were performed and appropriate blood work obtained.

Subjects received a $1 \mathrm{mg}$ intravenous bolus dose of $\left[{ }^{15} \mathrm{~N}_{3}\right.$ ]-labeled midazolam. Blood samples were col- 
lected at predose and 5, 10, 15, 30, 60, 90, 120, 180, 240, 300,360 , and 480 minutes following midazolam administration. Collected samples were centrifuged, and plasma was frozen at $-20^{\circ} \mathrm{C}$ until analysis.

\section{Analytical Procedure}

At Institutions I and III, midazolam assays were performed by liquid chromatography/tandem mass spectrometry (LC/MS/MS) methodology. This analytical procedure was performed at Oneida Research Services in Whitesboro, New York, for plasma samples collected at Institution I, ${ }^{8}$ and this analytical procedure had an interday accuracy of $-5.7 \%$ to $9.2 \%$ and precision of $9.9 \%$ over nominal values. The LC/MS/MS assay performed at Institution III had an interday variation of less than $12 \% .{ }^{19}$ Plasma samples were analyzed using electron capture gas chromatography at Institution II, a method previously published by Arendt et al. ${ }^{20}$

\section{Pharmacokinetic Analysis}

Midazolam $\mathrm{AUC}_{0-\infty}\left(\mathrm{AUC}_{\mathrm{obs}}\right)$ values were determined using the log-linear trapezoidal rule using the plasma concentration-time data, with extrapolation of the last measured concentration to infinity. Institution I used the pharmacokinetic computer program, TOPFIT 2.0 (Gustav Fisher Verlag, Stuttgart, Germany), for determining midazolam $\mathrm{AUC}_{0-\infty}$. Because plasma samples were not obtained at 5 minutes after midazolam administration at Institution II, the 5-minute plasma concentration was extrapolated for each subject using "back projection" or "stripping technique" principles. ${ }^{21}$ Pharmacokinetic parameters were determined using SAS PROC NLIN (SAS Institute, Cary, NC) at Institution II and the computer program NCOMP at Institution III. ${ }^{22}$ Elimination rate constants were calculated from using linear regression analysis of the terminal portion of the serum concentration versus time curve. All pharmacokinetic programs used for analysis are validated programs.

\section{Data Analysis}

Data were used to develop a training set (a set providing the best equations for prediction of AUC). The training set equations were applied to the remaining subject data to validate the prediction equations. All data were dose normalized to a dose of $1 \mathrm{mg}$.

Eleven randomly chosen subjects from Institution I were used as the training set. ${ }^{23}$ Using stepwise multiple linear regression, coefficients of determination $\left(r^{2}\right)$ were generated from the training set for one-, two-, and three-variable models to best predict $\mathrm{AUC}_{0-\infty}$ values $\left(\mathrm{AUC}_{\mathrm{pred}}\right)$ of midazolam.

The models were validated using the data from the remaining 61 subjects. Predicted AUC values $\left(\mathrm{AUC}_{\mathrm{pred}}\right)$ and observed $A U C$ values $\left(\mathrm{AUC}_{\mathrm{obs}}\right)$ were analyzed, and then AUC data were log transformed in all cases to normalize the distribution of the data. $\mathrm{AUC}_{\text {pred }}$ were compared with $\mathrm{AUC}_{\text {obs }}$ by calculating mean percent error (MPE) and mean absolute error (MAE) for precision and bias, using the following equations: ${ }^{24}$

$$
\begin{gathered}
\mathrm{MPE}=\sum_{\mathrm{i}=1}^{N}\left(\frac{\mathrm{AUC}_{\text {pred }}-\mathrm{AUC}_{\mathrm{obs}}}{n}\right), \\
\mathrm{MAE}=\sum_{\mathrm{i}=1}^{N}\left|\frac{\mathrm{AUC}_{\text {pred }}-\mathrm{AUC}_{\text {obs }} \mid}{n}\right| \\
\mathrm{RMSE}=\sqrt{(1 / n)\left(\sum_{\mathrm{i}=1}\left(\left|\mathrm{AUC}_{\text {pred }}-\mathrm{AUC}_{\mathrm{obs}}\right|^{2}\right)\right)} .
\end{gathered}
$$

Acceptable limits for precision and bias estimation for MPE, MAE, and RMSE were set at $<5 \%,<10 \%$, and $<$ $15 \%$, respectively.

Ten subjects in the validation set had five repeated administrations of single IV midazolam boluses at 2-week intervals. MPE, MAE, and RMSE were calculated to compare intraindividual variability to validate the reproducibility of the developed models.

To assess if the training set developed using the assay from Institution I was applicable to the assay methods from the other two institutions, data from the 40 subjects from Institutions II and III were analyzed using the training set to assess predictive ability. MPE, MAE, and RMSE were calculated to compare variability between institutions to validate the reproducibility of the developed models. Analyses were done using SAS V6.08 and MS Excel (Microsoft Corp., Redmond, WA). Data are presented as mean standard deviation $(S D)$, and $p$-values 0.05 were considered statistically significant.

\section{RESULTS}

Based on data from the training set, three different models with corresponding $r^{2}$ values were generated (Table I). The 300-minute plasma sample was the best one-variable model with an $r^{2}$ of 0.87 . The most accurate two-variable model used time points at 60 and 300 minutes $\left(r^{2}=0.95\right)$. The best three-variable model used 
Table I Summary of $r^{2}$ Values for Different Plasma Sampling Strategies for One, Two, and Three Midazolam Plasma Samples: Training Set (11 subjects)

\begin{tabular}{llll}
\hline \hline Model & Plasma Sampling Times (min) & \multicolumn{1}{c}{ Sampling Strategy for AUC pred } & \multicolumn{1}{c}{$\boldsymbol{r}^{2}$} \\
\hline 1 & 300 & $653.393 C_{300}+954.2257$ & 0.87 \\
2 & 360 & $968.526 C_{360}+855.8843$ & 0.78 \\
3 & 60,300 & $65.55 C_{60}+506.283 C_{300}+626.688$ & 0.95 \\
4 & 30,360 & $40.355 C_{30}+798.579 C_{360}+528.352$ & 0.93 \\
5 & $5,30,360$ & $12.265 C_{5}+24.0024 C_{30}+808.1387 C_{360}+430.4797$ & 0.95 \\
\hline
\end{tabular}

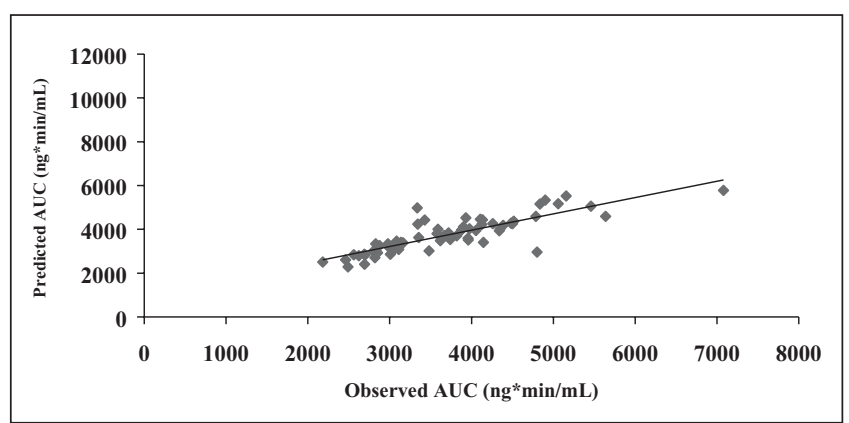

Figure 1. Predicted versus observed $A U C_{0-\infty}$ for plasma samples obtained at 300 minutes following midazolam administration. $\mathrm{C}_{\mathrm{n}}=$ midazolam concentration obtained at specified time n ( $\mathrm{min}$ ) following a single intravenous dose. $A U C_{0-\infty}=$ area under the curve extrapolating from the last measured concentration to infinity.

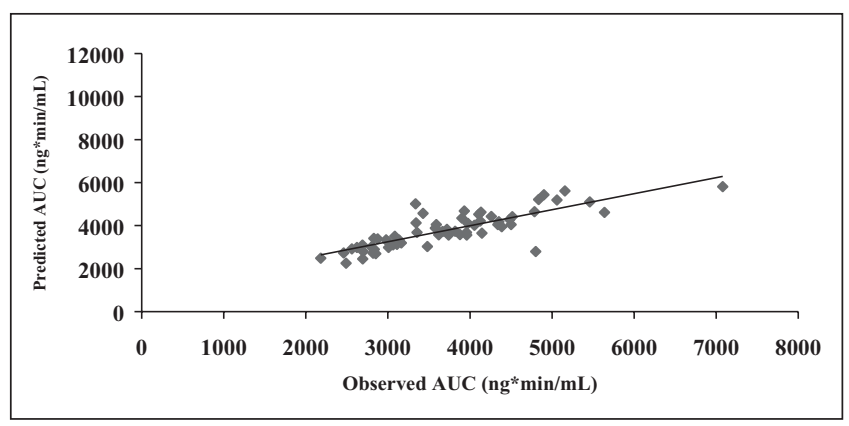

Figure 2. Predicted versus observed $A U C_{0-\infty}$ for plasma samples obtained at 60 and 300 minutes following midazolam administration. $\mathrm{C}_{\mathrm{n}}=$ midazolam concentration obtained at specified time $\mathrm{n}(\mathrm{min})$ following a single intravenous dose. $A U C_{0-\infty}=$ area under the curve extrapolating from the last measured concentration to infinity.

data from 5,30 , and 360 minutes $\left(r^{2}=0.95\right)$. Two additional one- and two-variable models are shown in Table I. Figures 1 through 3 graphically depict $r^{2}$ values for the

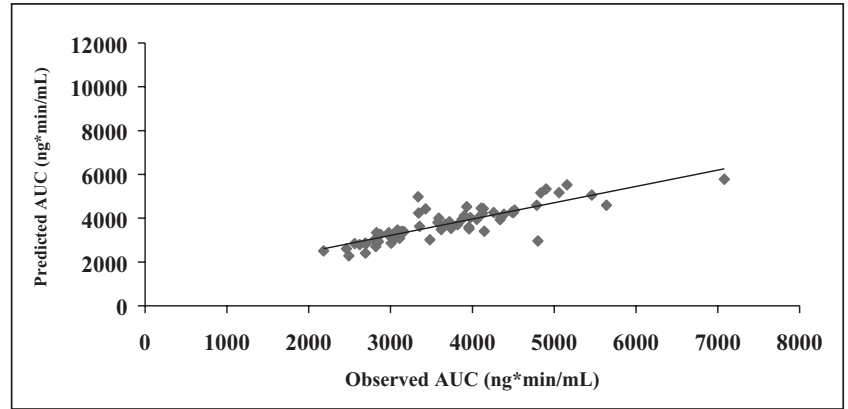

Figure 3. Predicted versus observed $A U C_{0-\infty}$ for plasma samples obtained at 5, 30, and 360 minutes following midazolam administration. $\mathrm{C}_{\mathrm{n}}=$ midazolam concentration obtained at specified time $\mathrm{n}$ (min) following a single intravenous dose. $A U C_{0-\infty}=$ area under the curve extrapolating from the last measured concentration to infinity.

best one-, two- and three- variable models by comparing $\mathrm{AUC}_{\mathrm{obs}}$ versus $\mathrm{AUC}_{\text {pred }}$.

Using the validation set, the percentages of mean percent error (MPE), mean absolute error (MAE), and root mean square error (RMSE) values were calculated for precision and bias, as shown in Table II. When $\mathrm{AUC}_{\text {pred }}$ and $\mathrm{AUC}_{\mathrm{obs}}$ data were normalized by log transformation, each sampling schema met acceptable precision and bias limits. The percentage errors were also calculated for five repeated visits at 2-week intervals for 10 subjects from Institution I to evaluate the reproducibility of errors using the models developed from the first visit data (see Table III). Data in Table III are for Visits 2 and 3. Results were similar for Visits 4, 5, and 6 (data not shown). Evaluation of the predictive equations showed good intrasubject reproducibility.

Table IV shows the results of the analysis using the training set data from Institution I to predict AUC from Institutions II and III. As noted in Table IV, good predic- 
Table II Percentage Errors Normalized via Log Transformation of $\mathrm{AUC}_{\text {pred }}$ and $\mathrm{AUC}_{\mathrm{obs}}$ for Precision and Bias for One, Two, and Three Midazolam Plasma Samples: Validation Set (62 subjects)

\begin{tabular}{|c|c|c|c|c|c|}
\hline Model (time points) & $\log A U C_{\text {pred }}$ & Range Log AUC ${ }_{\text {pred }}$ & $\%$ MPE & $\%$ MAE & \%RMSE \\
\hline $300 \min$ & $3.43 \pm 0.15$ & $3.14-3.7$ & 1.0 & 3.3 & 0.4 \\
\hline $60,300 \mathrm{~min}$ & $3.39 \pm 0.14$ & 3.14-3.65 & 0.1 & 1.5 & 1.8 \\
\hline $5,30,360 \mathrm{~min}$ & $3.45 \pm 0.16$ & $3.16-3.71$ & 1.7 & 2.9 & 4.8 \\
\hline Acceptable limits & & & $<5 \%$ & $<10 \%$ & $<15 \%$ \\
\hline
\end{tabular}

rum concentration time curve from 0 to infinity.

Table III Percentage Errors Normalized via Log Transformation for Precision and Bias for One, Two, and Three Midazolam Plasma Samples from Repeated Visits (2 and 3) for 10 Subjects

\begin{tabular}{lccc}
\hline \hline Time Postdose (min) & $\begin{array}{c}\text { Mean } \\
\text { Percent } \\
\text { Error }\end{array}$ & $\begin{array}{c}\text { Mean } \\
\text { Absolute } \\
\text { Error }\end{array}$ & $\begin{array}{c}\text { Root Mean } \\
\text { Square } \\
\text { Error }\end{array}$ \\
\hline Visit 2 & & & \\
$\quad 300$ & 5.0 & 5.0 & 5.5 \\
60, 300 & 6.8 & 6.8 & 7.5 \\
5, 30, 360 & 6.6 & 6.6 & 7.3 \\
Visit 3 & & & \\
300 & 3.1 & 3.8 & 4.3 \\
60, 300 & 6.4 & 6.4 & 7.0 \\
5, 30, 360 & 6.0 & 6.0 & 6.6 \\
Acceptable limit & $<5 \%$ & $<10 \%$ & $<15 \%$ \\
\hline
\end{tabular}

tive ability was seen using the training set from Institution I to predict data from the two other institutions and different assays.

\section{DISCUSSION}

This study was conducted to explore the possibility of applying LSS to CYP3A phenotyping with midazolam, validate this methodology with different assay techniques, and determine if the derived models were reproducible. Historically, LSS proved to be an effective tool in monitoring the safety and efficacy of chemotherapeutic agents by minimizing the number of blood samples necessary to evaluate AUC. ${ }^{25-28}$ AUC is a useful parameter that is quantitatively related to the myelosuppressive effects of these agents. Evaluation of such detailed pharmacokinetics requires multiple plasma sampling (e.g., 16 blood samples with
Table IV Percentage Errors Normalized via Log Transformation for Precision and Bias for One, Two, and Three Midazolam Plasma Samples Using Training Set (11 subjects from Institution 1) to Predict Data from 40 Subjects from Institutions II and III

\begin{tabular}{lccc}
\hline \hline Model (time points) & \%MPE & \%MAE & \%RMSE \\
\hline 300 min & 1.4 & 4.2 & 5.8 \\
60, 300 min & 0.2 & 0.2 & 2.0 \\
5, 30, 360 min & 2.5 & 3.7 & 5.8 \\
Acceptable limits & $<5 \%$ & $<10 \%$ & $<15 \%$ \\
\hline \multicolumn{2}{l}{ MPE, mean percent error; MAE, mean absolute error; RMSE, root mean } \\
square error; AUC, area under the serum concentration time curve from 0 to \\
infinity.
\end{tabular}

vinblastine), which is usually not feasible in large numbers of patients.

Using a limited number of samples to evaluate the pharmacokinetic profile of agents is more attractive than multiple plasma sampling. Scavone et $\mathrm{al}^{13}$ validated the use of two points at 2 and 6 hours versus 3 and 6 hours to assess the elimination half-life $(r=0.87$ and $r=0.84)$ and clearance $(r=0.97$ and $r=0.97)$ of acetaminophen. That study, however, overestimated clearance and volume of distribution by approximately $13 \%$ and $14 \%$. The authors concluded that distribution must have contributed significantly to total AUC, as previously shown in an earlier study with antipyrine. ${ }^{12}$

The method of minimized plasma sampling uses stepwise linear regression to determine optimal concentration time points in a model derived from a training data set to accurately predict pharmacokinetic parameters such as AUC. The utility of the LSS method was first illustrated with vinblastine. With only two time points, Ratain and Vogelzang ${ }^{10}$ were able to successfully estimate total AUC with minimal error using stepwise multiple regression analysis. The correlation 
coefficient was $>0.85$ at time points of 1 hour or later following vinblastine infusion, whereas only a modest correlation was demonstrated for plasma samples obtained between 5 and 20 minutes $(r<0.58)$. The two best correlation coefficients $(r=0.97)$ were at 10 hours and 36 hours. The model was highly predictive of AUC, illustrated by the predictive error of $13 \%$. Other drugs for which minimized plasma sampling has proven effective at predicting AUC include cyclophosphamide, doxorubicin, etoposide, carboplatin, irinotecan, antipyrine, and acetaminophen. ${ }^{11-13,25-27,29-32}$ Similar success has been found in predicting AUC by using limited plasma sampling to monitor the efficacy and toxicity profiles of various immunosuppressives, such as mycophenolic acid and cyclosporine. ${ }^{33-35}$

Validation of the study models was accomplished by calculating percentage errors. Percentage errors, such as mean percent error and mean absolute error, provide information about the precision and bias of the predictive value $\left(\mathrm{AUC}_{\text {pred }}\right)$ when using derived models to estimate AUC. Absolute errors (i.e., MAE) represent the precision of the predicted value. The mean percentage errors (i.e., MPE) describe the prediction bias. ${ }^{24}$ These values can serve as a tool to assess the model that predicts the mathematically derived parameter with the most precision and the least bias (the error percentages with the lowest values represent the best models). Generally, the limits for these errors are not specific values for each study, and the majority of limited sampling studies report error percentages that are $<5 \%$ for MPE, $<$ $10 \%$ for MAE, and $<15 \%$ for RMSE. ${ }^{36,37}$

This study examined the use of minimized plasma sampling with intravenous midazolam. Three of the models generated from our training set using one-, two-, or three-plasma samples provided $r^{2}$ values of 0.87. The two- and three-variable models illustrated the best $r^{2}$ value $\left(r^{2}=0.95\right)$. When the AUC data were normalized by log transformation, each model showed excellent precision with minimal bias. Although all models perform well, the three-point model will perform best in instances when an assay measurement may be erroneous since the erroneous point will become evident.

The one-, two-, and three-variable models validated in 62 subjects were also validated in a subset of these subjects $(n=10)$ to evaluate the reproducibility of the derived models to the same individuals at different time intervals. The models projected acceptable mean values for MPE, MAE, and RMSE, respectively. These models, therefore, expressed acceptable intrasubject reproducibility.
Another question is whether or not training models produced using data from one institution or assay method can be used to predict data from other institutions. The one-, two-, and three-variable models developed in 11 subjects from Institution I were evaluated in the 40 subjects from Institutions II and III to assess the reproducibility of the models. The models projected acceptable mean values for MPE, MAE, and RMSE, respectively.

A limitation of this study was that a small sample size of healthy volunteers was used to develop the models and may not reflect ascertainment of AUC over the entire population or in nonhealthy individuals. These models are also not applicable to pediatric subjects as children exhibit different midazolam pharmacokinetic parameters from adults. ${ }^{38}$ Finally, the schedules for blood sampling differed somewhat among institutions, compromising the accuracy for predicted AUC values. Further prospective studies with larger numbers and different types of individuals are needed to better characterize these models.

A strength of this study is that it validated models developed by one institution using one assay method with plasma samples quantified by different assay techniques. This validation indicates that these models may be used in institutions that use various assay techniques. Most minimized sampling studies use a single assay method or do not report the types of assay techniques used in the studies. In the current study, the models derived from one assay technique were applicable to different assays, making these models more universal to other institutions.

Minimized plasma sampling provides an accurate and precise method for evaluating midazolam AUC in adults. The use of LSS may be a more convenient, cost-effective, and useful method for measuring hepatic CYP3A activity with midazolam.

\section{REFERENCES}

1. Tucker GT, Rostami-Hosdjegan A, Jackson PR: Determination of drug-metabolizing enzyme activity in vivo: pharmacokinetic and statistical issues. Xenobiotica 1998;28:1255-1273.

2. Streetman DS, Bertino JS Jr, Nafziger AN: Phenotyping of drugmetabolizing enzymes in adults: a review of in-vivo cytochrome P450 phenotyping probes. Pharmacogenetics 2000;10:187-216.

3. Thummel KE, Shen DD, Podoll TD, Kunze KL, Trager WF, Hartwell PS, et al: Use of midazolam as a human cytochrome P450 3A probe: I. In vitro-in vivo correlations in liver transplant patients. J Pharmacol Exp Ther 1994;271:549-556.

4. Thummel KE, Shen DD, Podoll TD, Kunze KL, Trager WF, Bacchi $\mathrm{CE}$, et al: Use of midazolam as a human cytochrome P450 3A probe: II. Characterization of inter- and intraindividual hepatic CYP3A vari- 
ability after liver transplantation. J Pharmacol Exp Ther 1994: 557-566.

5. Thummel KE, O'Shea DD, Paine MF, Shen DD, Kunze KL, Perkins JD, et al: Oral first-pass elimination of midazolam involves both gastrointestinal and hepatic CYP3A-mediated metabolism. Clin Pharmacol Ther 1996;59:491-502.

6. Tsunoda SM, Velez RL, von Moltke LL, Greenblatt DJ: Differentiation of intestinal and hepatic cytochrome P450 3A activity with the use of midazolam as an in vivo probe: effect of ketoconazole. Clin Pharmacol Ther 1999;66:461-471.

7. Kashuba ADM, Nafziger AN, Kearns GL, Leeder JS, Gotschall R, Rocci ML, et al: Effect of fluvoxamine therapy on the activities of CYP1A2, CYP2D6, and CYP3A as determined by phenotyping. Clin Pharmacol Ther 1998;64:257-268.

8. Kashuba ADM, Bertino JS, Rocci ML, Kulawy RW, Beck DJ, Nafziger AN: Quantification of 3-month intraindividual variability and the influence of sex and menstrual cycle phase on CYP3A activity as measured by phenotyping with intravenous midazolam. Clin Pharmacol Ther 1998;64:269-277.

9. Greenblatt DJ, Abernethy DR, Locniskar A, Harmatz JS, Limjuco RA, Shader RI: Effect of age, gender, and obesity on midazolam kinetics. Anesthesiol 1984;61:27-35.

10. Ratain MJ, Vogelzang NJ: Limited sampling model for vinblastine pharmacokinetics. Cancer Treat Rep 1987;71:935-939.

11. Ratain MJ, Robert J, van der Vijgh WJF: Limited sampling models for doxorubicin pharmacokinetics. J Clin Oncol 1991;9:871-876.

12. Scavone JM, Greenblatt DJ, Blyden GT, Harmatz JS, Graziano PJ: Simplified approaches to the determination of antipyrine pharmacokinetic parameters. Br J Clin Pharmacol 1988;25:695-699. 13. Scavone JM, Greenblatt DJ, Blyden GT, Luna BG, Harmatz JS: Validity of a two-point acetaminophen pharmacokinetic study. Ther Drug Monit 1990;12:35-39.

14. Charlebois JE, Lum BL, Cooney GF, Mochon M, Kaiser BA: Comparison and validation of limited sampling equations for cyclosporine area-under-the-curve monitoring calculations in pediatric renal transplant recipients. Ther Drug Monit 1997;19:277-280. 15. Chattergoon DS, Saunders EF, Klein J, Calderwood S, Doyle J, Freedman MH, et al: An improved limited sampling method for individualized busulphan dosing in bone marrow transplantation in children. Bone Marrow Transplant 1997;20:347-354.

16. Eksborg S, Albertioni F, Beck O, Peterson C, Seideman P: Methotrexate in rheumatoid arthritis: a limited sampling strategy for estimation of the area under the plasma concentration versus time curve. Ther Drug Monit 1994;16:560-563.

17. Suarez-Kurtz G, Bozza FA, Vicente FL, Ponte CG, Struchiner CJ, et al: Limited-sampling strategy models for itraconazole and hydroxy-itraconazole based on data from a bioequivalence study. Antimicrob Agents Chemo 1999;43:134-140.

18. Streetman DS, Bleakley JF, Kim JS, Nafziger AN, Leeder JS, Gotschall R, et al: Validation of a four-drug phenotyping cocktail (PC). Clin Pharmacol Ther 2000;67:150.

19. Wandel C, Witte JS, Hall JM, Stein CM, Wood AJJ, Wilkinson GR: CYP3A activity in African- and European-American men: population differences and functional effect of the CYP3A4*1B 5'-promoter region (NFSE) polymorphism. Clin Pharmacol Ther 2000;68:82-91.

20. Arendt RM, Greenblatt DJ, Garland WA: Quantitation by gas chromatography of the 1- and 4-hydroxy metabolites of midazolam in human plasma. Pharmacology 1984;29:158-164.
21. Wagner JG: Linear compartment models, in: Wagner JG (ed.), Fundamentals of Clinical Pharmacokinetics. Hamilton, IL: Drug Intelligence Publications, 1975;59-62.

22. Laub PB, Gallo JM: NCOMP: a Windows-based computer program for noncompartmental analysis of pharmacokinetic data. $J$ Pharm Sci 1996;85:393-395.

23. Kim JS, Kashuba ADM, Nafziger AN, Rocci ML, Kulawy RW, Beck DJ, et al: Optimal plasma sampling to predict AUC of the CYP3A probe midazolam (MID). Clin Pharmacol Ther 1999;65:185.

24. Sheiner LB, Beal SL: Some suggestions for measuring predictive performance. J Pharmacokin Biopharm 1981;9:503-512.

25. Egorin MJ, Forrest A, Belani CP, Ratain MJ, Abrams JS, Van Echo DA: A limited sampling strategy for cyclophosphamide pharmacokinetics. Cancer Res 1989;49:3129-3133.

26. Chabot GG: Limited sampling models for simultaneous estimation of the pharmacokinetics of irinotecan and its active metabolite SN-38. Cancer Chemother Pharmacol 1995;36:463-472.

27. Doz F, Urien S, Chatelut E, et al: A limited-sampling method for evaluation of the area under the curve of ultrafilterable carboplatin in children. Cancer Chemother Pharmacol 1998;42:250-254.

28. Holz JB, Koppler H, Schmidt L, Fritsch HW, Pfluger KH, Jungclas $\mathrm{H}$ : Limited sampling models for reliable estimation of etoposide area under the curve. Eur J Cancer 1995;31A:1794-1798.

29. Miyazaki M, Fujiwara $Y$, Takahashi T, Isobe T, Ohune T, Tsuya T, et al: Limited-sampling models for estimation of the carboplatin area under the curve. Anticancer Res 1997;17(6D):4571-4575.

30. Gentili D, Zucchetti M, Torri V, Sessa C, de Jong J, Cavalli F, et al: A limited sampling model for the pharmacokinetics of etoposide given orally. Cancer Chemother Pharmacol 1993;32:482-486.

31. Sorensen BT, Stromgren A, Jakobsen P, Jakobsen A: A limited sampling method for estimation of the carboplatin area under the curve. Cancer Chemother Pharmacol 1993;31:324-327.

32. Stromgren AS, Sorensen BT, Jakobsen P, Jakobsen A: A limited sampling method for estimation of the etoposide area under the curve. Cancer Chemother Pharmacol 1993;32:226-230.

33. Schutz E, Armstrong VW, Shipkova M, Weber L, Niedmann PD, Lammersdorf T, et al: Limited sampling strategy for the determination of mycophenolic acid area under the curve in pediatric kidney recipients. German Study Group on MMF Therapy in Pediatric Renal Transplant Recipients. Transplant Proc 1998;30:1182-1184.

34. Johnston A, Sketris I, Marsden JT, Galustian CG, Fashola T, Taube D, et al: A limited sampling strategy for the measurement of cyclosporine AUC. Transplantation Proc 1990;22:1345-1346.

35. Serafinowicz A, Gaciong Z, Baczkowska T, Rell K, Lao M, Walsazewski J, et al: Limited sampling strategy to estimate exposure to cyclosporine A in renal allograft recipients treated with Sandimmune-Neoral. Transplant Proc 1996;28:3138-3139.

36. Mahmood I: A comparative computer simulation study of three different sparse-sampling methods for the estimation of steady-state area under the concentration-time curve (AUC) and maximum concentration $\left(\mathrm{C}_{\max }\right)$ in toxicokinetics. J Pharm Sci 1996;86:579-583.

37. Mahmood I: Development of a limited sampling approach in pharmacokinetic studies: experience with the antiepilepsy drug tiagabine. J Clin Pharmacol 1998;38:324-330.

38. Burtin P, Jacqz-Aigrain E, Girard P, Lenclen R, Magny JF, Betremieux P, et al: Population pharmacokinetics of midazolam in neonates. Clin Pharmacol Ther 1994;56:615-625. 